\title{
小学数学错题资源的再利用研究
}

侯林光

山西省大同市实验小学文博分校

DOI:10.32629/jief.v2i4.1275

[摘 要] 本文分析了在小学数学教学中学生经常做错题的原因, 主要是因为学生没有理解概念和规律, 或者缺乏审题能力, 以及想当然。提 出在教学中进行错题资源再利用的方法, 即找出学生做错题的具体原因, 对错题加以巧妙利用, 以及在出题时科学地设置陷吽, 以检查学生 是否彻底掌握了错题的知识点。

[关键词] 小学数学; 错题; 资源再利用

中图分类号：G633.6 文献标识码：A

在小学数学教学中, 学生做错题是一种经常出现的情况, 也是非常正常 的教学情况。有些数学教师不重视错题的价值, 对错题进行讲解后就抛到一 边, 这实际上是一种资源浪费。合理利用错题, 将错题整理编辑成册, 有利 于在教师后续的教学工作中作为参考, 有针对性地对学生进行习题训练。

\section{1 错题产生的原因}

1.1 没有理解概念和规律

小学数学的知识架构相对而言比较单一, 多以概念、规律和一些联系 生活实际的基础性问题为主。小学生需要解答的数学问题, 一般都是从这 些基础性的概念或者规律出发, 围绕知识点进行题目设置。学生做错题, 是因为对知识点掌握不牢, 对这些概念和规律没有理解透彻。只有学生充 分掌握了知识点, 做到对知识点的深入理解, 才能在做题时运用知识点解 答问题。如果只是死记硬背而不理解, 那么就会重复出现错误。

1.2 缺乏审题能力

除了对知识点掌握的程度不高, 还有一种情况也会导致学生做错题, 那就是学生没有读懂题目。如果学生连题目中说了什么内容都不理解, 自然也就无从解起。培养学生的数学能力, 首先就要求学生要学会审题, 审清题目后, 知道题目说了什么, 要求解的问题是什么, 才能对题目进 行下一步的分析, 然后才能解答。教师在课堂教学时, 要教导学生在审 题时分清逻辑关系, 抓住题目重点, 找出与问题有关的关键条件, 只有 这样, 才能明确审题, 正确解答问题。

\section{3 读题潦草，做题想当然}

有些学生性格毛躁, 做题马虎, 在读题时常常不够认真, 粗略读了 一遍题目以后就想当然地按照惯性思维进行解题。小学生在学习过程中 容易被思维定势所影响, 由既有的知识内容无意识地派生出不一定正确 的新知识。在教学过程中, 教师要随时关注这方面的问题, 对学生进行 及时有效的纠偏。

\section{2 错题资源的再利用方法}

2.1 找出错误原因

数学教师在发现学生做错题以后, 首先应该认识到学生做错题是必 然会发生的情况, 不必为此焦急甚至是生气。小学生处于心理发展阶段, 对老师的情绪变化很敏感。如果这样的情况发生, 不利于师生之间建立 健康的教学关系, 不仅会给学生学习造成困难, 也会阻碍教学。教师应 该意识到, 出现问题就是解决问题的契机, 要抓住这个契机, 发现学生 学习中的薄弱点, 目标明确地进行解决。教师应全面分析错题出现的原 因, 研究是哪一个知识点没有被学生掌握。例如, 在学习平方时, 教师 在黑板上写下:

$3^{3}=$ ?

提问后, 学生回答错误。这个时候老师不必急于纠正, 而是应该进 行进一步引导, 告诉学生, 虽然看起来这是两个一模一样的 “ 3 ”, 但是
这前后两个 “ 3 ” 代表的涵义却不同, 第二个 “ 3 ” 代表的是立方。学生 就会领悟问题出在哪里, 给出正确的答案。利用这种比较有代表性的错 题, 可以作为对学生的提醒。

\section{2 巧妙利用错题}

在教学实践中, 不仅要注意对错题进行纠正, 还要对错题进行巧妙 地利用。尤其是以生活中的情景出题, 让学生们发现自己的答案存在逻 辑上的错误。利用生活中的例子, 是小学数学教学中经常会用到的方法, 既联系了实际, 使学生们体会到数学的应用意义, 提升了学生学习数学 的兴趣, 也可以用现实中的逻辑, 使学生意识到在做数学题时必须要有 灵活性。例如, 教师出了这样一道题:

小明要去宠物店买圆珠笔, 已知小明有 110 元钱, 圆珠笔 6 元一支, 问: 小明最多能买多少支圆珠笔?

学生在解答过程中会发现无法被整除, 就会产生疑惑, 认为自己算错 了, 或者是老师出错题了。这时候, 老师就应该引导学生, 如果你是小明, 售货员会卖给你几支圆珠笔? 经过循序渐进的引导, 学生就会明白在解答 数学题时, 不仅要做到运算准确, 还应该联系实际情况进行解答。

2.3 科学设置陷阴

陷阱题也是学生经常会做错的题。小学生思想比较单纯, 对于题目中 故意设置的陷阱, 往往不能察觉, 而是被陷阱引导, 给出错误的答案。学 生出现这种错题情况, 就要求教师加强陷阱题的训练, 使学生明白陷阱的 原理, 在做题时提高警惕, 避免思路被陷阱带偏。教师在教学中, 应该主 动加强对学生的陷阱题训练, 使学生在解决数学题时更加敏锐。例如:

小明家距离小强家 300 米, 小强到小刚家 200 米, 如果小明想去小 刚家, 需要走多少米?

学生看到这道题后, 往往会想当然地回答 “500 米”, 这时候老师就 要说明, 三人的位置并不确定, 所以答案有两个。

综上所述, 错题其实是一种非常有利用价值的资源。充分利用错题 集, 可以帮助数学教师总结学生容易出错的知识点, 了解容易对学生造 成误导的问题陷阱。对错题集进行充分而科学的再利用, 可以有效提高 学生的知识掌握水平, 提高课堂的教学质量, 使小学数学教学工作得到 事半功倍的效果。

\section{[参考文献]}

[1]陈国付.小学高年级数学错题本建立与有效利用分析 [J].学周 刊,2020(05):68.

[2]冯德军. 小学数学错题资源的再利用时机分析 [J]. 课程教育研 究,2019(51):129-130.

[3] 陈再明. 有效构建小学数学互动课堂策略 [N]. 贵州民族 报,2020-08-27(B03). 